\title{
Ultrasound-assisted synthesis of 2,4-thiazolidinedione and rhodanine derivatives catalyzed by task-specific ionic liquid: [TMG][Lac]
}

\author{
Suresh $^{*}$ and Jagir Singh Sandhu
}

\begin{abstract}
Background: Synthesized arylidene derivatives of rhodanine and 2,4-thiazolidiendione have potent pharmacological activities, and these are also key substrates for the preparation of clinically used antidiabetics.

Findings: Some 1,1,3,3-tetramethylguanidine-based task-specific ionic liquids (TSILs) 1a-1e were prepared and employed to the catalyzed solvent-free Knoevenagel condensation of 2,4-thiazolidinedione $3 \mathrm{a}$ and rhodanine $3 \mathrm{~b}$ with a variety of aldehydes.

Conclusions: Best results were obtained with 1,1,3,3-tetramethylguanidine lactate ([TMG][Lac]) 1c. The TSIL used can be easily recovered and recycled, yielding products $4-5$ in excellent yields under ultrasonic environment without the formation of any side products or toxic waste.
\end{abstract}

Keywords: 2,4-thiazolidinedione, Rhodanine, Knoevenagel condensation, Recyclability, Task-specific ionic liquid

\section{Findings}

\section{Background}

2,4-thiazolidenedione (TZD) is an attractive scaffold because of its prestigious position in medicinal chemistry as this unit is responsible for numerous pharmacological and biological activities, e.g., antidiabetic [1,2], antidiarrheal [3], anticonvulsant [4], antimicrobial [5], antihistaminic [6], anticancer [7], anti-HIV [8], 15-hydroxyprostaglandin dehydrogenase inhibitors [9], and anti-ischemic [10]. The position of these molecules seems to be most significant as they are a subset of commercially employed non-insulindependent diabetes mellitus and insulin-sensitizing agents (Figure 1) such as rosiglitazone, epalrestat, ciglitazone, AD5061, pioglitazone, and so on.

Several methods have been developed for their condensation with aldehydes as this is a crucial step in the production of the above clinically used drugs (Figure 1), and thus, this condensation is of commercial value. To achieve this end, several production protocols are reported employing several catalysts used such as amines [11], amine derivatives [12], amines salts [13], baker's yeast [14], sodium acetate [15-17], glycine [18,19], $\mathrm{KF}-\mathrm{Al}_{2} \mathrm{O}_{3}$

\footnotetext{
* Correspondence: jind_suresh7@rediffmail.com

Department of Chemistry, Punjabi University, Patiala, Punjab 147 002, India
}

[20], and ionic liquids [21-25]. Several of these existing protocols for achieving this step have drawbacks like having long reaction times and low yields and leaving toxic residues on aqueous work-up; amine use is also now found to be carcinogenic. In addition, ionic liquids used earlier to accelerate this reaction are not environmentally benign because their preparation involves the harmful chemical intake, that is, volatile solvent and corrosive/toxic reagents [21-25]. Hence, a facile efficient process is still desirable. In search of greener chemical process development, ionic liquids are finding a prominent place [26-30]; herein, we report the first-ever sonically enhanced procedure using the environmentally benign ionic liquid 1,1,3,3-tetramethylguanidine lactate ([TMG][Lac]).

This ionic liquid based on TMGs is stable and easy to prepare in the laboratory, e.g., [TMG][Lac] 1c is prepared by neutralizing TMG with lactic acid [31]. [TMG] [Lac] has already been reported to accelerate important reactions such as Henry reactions, hydrogenation of olefins, hydrogenolysis of glycerol, aldol reaction, and so on [32-38]. Our own interest in ionic liquids (ILs) and in green synthetic transformations $[29,30]$ prompted us to explore the catalytic activities of TMG-based ILs in the synthesis of 2-TZD and rhodanine derivatives. On top of 


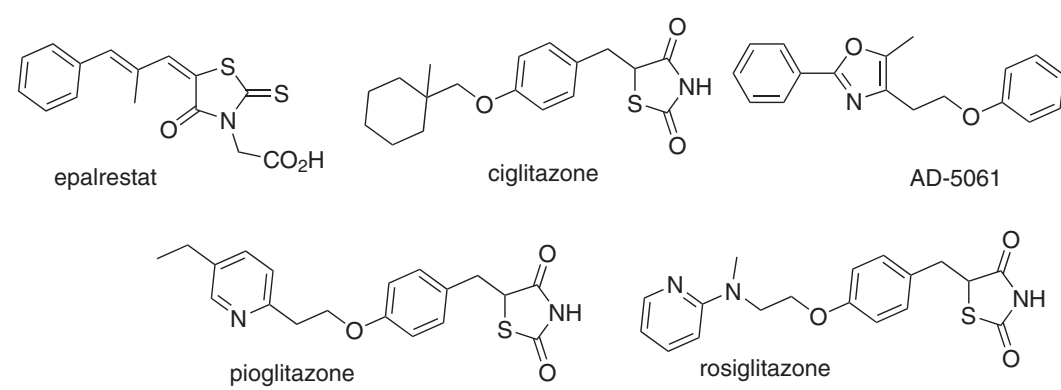

Figure 1 Clinically used molecules having 5-arylidene rhodanines and 2,4-thiazolidenediones.

all these factors, sonication is finding extensive use as it saves time and is environmentally friendly [39-41].

\section{Results and discussion}

In our initial pilot experiments, ionic liquids 1a-1e based on TMGs were used to catalyze condensation of 4-methoxybenzaldehyde $\mathbf{2 a}$ with 2,4-thiazolidinedione $\mathbf{3 a}$ (Table 1). The best results were obtained when the reaction was catalyzed by task-specific ionic liquid (TSIL) [TMG][Lac] 1c under ultrasound irradiations at $80^{\circ} \mathrm{C}$ for $20 \mathrm{~min}$ (Table 1 , entry 3) among the ILs 1a-1e. The amount of catalyst 1c was also established as $20 \mathrm{~mol} \%$ by carrying out reactions of 2a with 3a using different amounts of catalyst (5 to $50 \mathrm{~mol} \%$ ).

To check the effect of catalyst, temperature, and ultrasound irradiations, a set of reactions was performed using different reaction conditions such as heating alone (Table 2, entry 2), ultrasound alone (Table 2, entries 6 and 7), heating along with ultrasound irradiations (Table 2, entries 5 to 10), and in the absence of both (Table 2, entry 1). Conclusively, catalyst, temperature, and ultrasound irradiations are all equally important to accelerate the Knoevenagel condensation of arylaldehydes 2 and 2,4-thiazolidenedione/rhodanine 3 to afford 5-arylidene-2,4-thiazolidinediones and 5-arylidenerhodanines 4-5 with excellent yields (Table 2, entry 9).

Further, a variety of carbonyl compounds such as aromatic aldehydes $\mathbf{2 a}-\mathbf{2} \mathbf{b}$, heterocyclic aldehydes $\mathbf{2 c}-\mathbf{2 e}$ (furan-2-carbaldehyde 2c, thiophene-2-carbaldehyde 2d, and 3-formylchromone 2e) were condensed with 5-membered active hydrogen compounds 2,4-thiazolidinedione $\mathbf{3 a}$ and rhodanine $\mathbf{3 b}$ in the presence of $20 \mathrm{~mol} \% \mathbf{1 c}$ under ultrasound irradiations at $80^{\circ} \mathrm{C}$ under solvent-free conditions to provide Knoevenagel products $4-5$ in excellent yields (Scheme 1). Aromatic aldehydes $\mathbf{2 a - 2 b}$ afforded excellent yields in a shorter reaction time, whereas heterocyclic $2 \mathbf{c}-2 \mathbf{e}$ gave high yields in a slightly long reaction time (Table 3).

Active methylene compounds $\mathbf{3} \mathbf{a}-\mathbf{3 b}$ afforded the Knoevenagel products selectively with exo-double bond without the formation of other side products/bis-products as shown in Scheme 2. Electron-withdrawing and electron-donating groups on aromatic aldehyde showed a slight diversion in the rate of reaction and yields, i.e.,

Table 1 Synthesis of (Z)-5-(4-methoxybenzylidene)thiazolidine-2,4-dione 4a via Knoevenagel condensation of 2a with 3a employing different ILs as catalyst<smiles>COc1ccc(/C=C2\SC(=O)NC2=O)cc1</smiles>

\begin{tabular}{|c|c|c|c|}
\hline & & & \\
\hline Entry & ILs (20 mol\%) & Time (min) & Yield (\%) \\
\hline 1 & {$[\mathrm{TMG}]\left[\mathrm{CH}_{3} \mathrm{COO}^{-}\right]: \mathbf{1 a}$} & 50 & 92 \\
\hline 2 & {$[\mathrm{TMG}]\left[\mathrm{CF}_{3} \mathrm{COO}^{-}\right]: \mathbf{1 b}$} & 120 & 85 \\
\hline 3 & {$[\mathrm{TMG}]\left[\mathrm{CH}_{3} \mathrm{CHOHCOO}^{-}\right]: \mathbf{1 c}$} & 20 & 95 \\
\hline 4 & {$[\mathrm{TMG}]\left[\mathrm{CH}_{3} \mathrm{CH}_{2} \mathrm{COO}^{-}\right]: \mathbf{1 d}$} & 75 & 91 \\
\hline 5 & {$[\mathrm{TMG}]\left[\left(\mathrm{CH}_{3}\right)_{2} \mathrm{CH}_{2} \mathrm{COO}^{-}\right]: \mathbf{1 e}$} & 40 & 94 \\
\hline
\end{tabular}

The reaction conditions for (Z)-5-(4-methoxybenzylidene)thiazolidine-2,4-dione $\mathbf{4 a}$ are as follows: $\mathbf{2 a}$ (1 $\mathrm{mmol}), \mathbf{3 a}(1 \mathrm{mmol})$, and catalysts (20 mol\%) were irradiated under ultrasound irradiations at $80^{\circ} \mathrm{C}$. The product was characterized by spectral techniques like IR, ${ }^{1} \mathrm{H} N M R,{ }^{13} \mathrm{C}$ NMR, and mass spectra. ${ }^{\mathrm{a}}$ Isolated yields after recrystallization. 
Table 2 Condensation of $2 a$ and $3 a$ in the presence of different reaction conditions

\begin{tabular}{|c|c|c|c|}
\hline Entry & Catalyst & Time $(\min )$ & Yield $^{\mathrm{a}}$ \\
\hline 1 & No catalyst + No Heating + No US & 180 & - \\
\hline 2 & No catalyst + Heating $\left(50^{\circ} \mathrm{C}\right)$ & 180 & - \\
\hline 3 & Catalyst $(20 \mathrm{~mol} \%)+$ Heating $\left(50^{\circ} \mathrm{C}\right)$ & 60 & $<55$ \\
\hline 4 & Catalyst $\left(20\right.$ mol\%) + Heating $\left(80^{\circ} \mathrm{C}\right)$ & 60 & 68 \\
\hline 5 & No catalyst + Heating $\left(50^{\circ} \mathrm{C}\right)+$ US & 120 & $<10$ \\
\hline 6 & Catalyst (20 mol\%) + No Heating + US & 50 & 74 \\
\hline 7 & Catalyst (50 mol\%) + No Heating + US & 50 & 72 \\
\hline 8 & Catalyst $(20 \mathrm{~mol} \%)+$ Heating $\left(50^{\circ} \mathrm{C}\right)+$ US & 40 & 80 \\
\hline 9 & Catalyst $\left(20\right.$ mol\%) + Heating $\left(80^{\circ} \mathrm{C}\right)+$ US & 20 & 95 \\
\hline 10 & Catalyst $(20$ mol\% $)+$ Heating $\left(100^{\circ} \mathrm{C}\right)+$ US & 30 & 92 \\
\hline
\end{tabular}

The different reaction conditions are as follows: $1 \mathbf{a}(1 \mathrm{mmol}), \mathbf{2 a}(1 \mathrm{mmol})$, and catalysts were irradiated under ultrasound irradiations (US) at different temperatures. ${ }^{a}$ Isolated yield.

the electron-withdrawing group containing aromatic aldehydes afforded arylidene compounds 4-6 with better yields in a shorter reaction time (Table 3).

Next, the recyclability of the catalyst $\mathbf{1 c}$ was studied using 2a and 3a as the model substrate. We observed that $1 c$ could be recovered by extraction of the filtrate with a particular solvent, and pure IL (colorless oil) was obtained after drying the water-rich layer under vacuum. The recovered catalyst was successfully recycled and reused for five runs.

As plausible reaction mechanism is concerned, $\mathbf{1 c}$ catalyzed synthesis of arylidene products $\mathbf{4}-\mathbf{5}$ by facilitating the nucleophilic attack of 3a-3b (lactate anion abstract the active hydrogen of 3 ) on the electrophilic carbon of carbonyl compounds (activated by TGA cation) 2a-2e, and subsequent dehydration leads to the product formation (Scheme 2).

\section{Conclusion}

In summary, we have disclosed a clean and efficient procedure for the synthesis of pharmacologically significant thiazolidine derivatives via Knoevenagel condensation of aldehydes with 2,4-thiazolidinedione/rhodanine.
The task-specific ionic liquid [TMG][Lac] catalyst used is biodegradable, recyclable, and purely environmentally benign as it is easy to prepare, without the involvement of any harmful solvents/chemicals [31]; hence, it is better than already used imidazolium-based ionic liquids [21-25] as catalysts. The scope is fairly large as the range of aldehydes and active methylene compounds used afforded products in very good to excellent yields. Other prominent features are as follows: the reaction time is shorter, no polluting volatile solvents are used, reaction work-up is simple, no toxic by-products are formed during aqueous work-up, and all these green aspects place this method at an advantageous position compared to already reported methods for these molecules of commercial value.

\section{Methods}

General

All starting materials were commercial products and were used without further purification except liquid aldehydes, which were distilled before use. Yields refer to yield of the isolated products. Melting points were determined in open capillaries in a paraffin bath and are uncorrected. Nuclear magnetic resonance spectra were obtained on a $400 \mathrm{MHz}$ Bruker AMX instrument (Bruker Corporation, Billerica, MA, USA) in DMSO- $\mathrm{d}_{6}$ using TMS as a standard. HRMS analyses were carried out using a ESI-Q TOF instrument (Bruker Corporation). Infrared spectra were recorded using a Shimadzu FT-IR-8400 s spectrophotometer (Shimadzu Corporation, Kyoto, Japan) as $\mathrm{KBr}$ pellets. All the reactions were studied using a SIDILU Indian-made sonic bath (Sidilu Ultrasonic Technology, Bangalore India) working at $35 \mathrm{kHz}$ (constant frequency, $120 \mathrm{~W}$ ) maintained at $80^{\circ} \mathrm{C}$ without mechanical stirring.

\section{General procedure for the synthesis of arylidene- thiazolidenes}

A mixture of aldehyde (1 mmol), 2,4-thiazolidinedione/ rhodanine (1 mmol), and [TMG][Lac] $(20 \mathrm{~mol} \%)$ was irradiated under ultrasonic irradiation at $80^{\circ} \mathrm{C}$ for a few

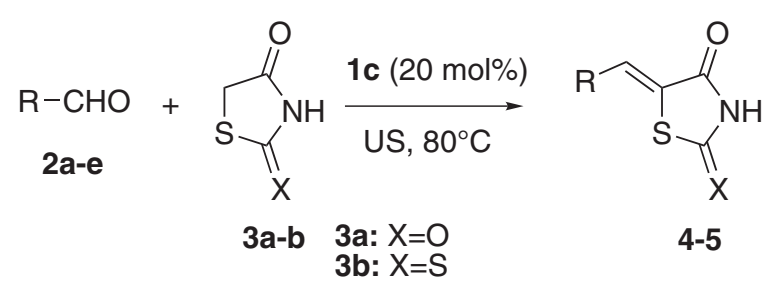

2a: $\mathrm{R}=4-\mathrm{MeO}-\mathrm{C}_{6} \mathrm{H}_{4}, 2 \mathrm{~b}: \mathrm{R}=4-\mathrm{Cl}-\mathrm{C}_{6} \mathrm{H}_{4}, 2 \mathrm{c}: \mathrm{R}=2$-furyl,

2d: $R=2$-thiophene, 2e: $R=3$-formylchromone

Scheme 1 Knoevenagel reaction of 2 and 3 catalyzed by $1 c$. 
Table 3 [TMG][Lac]-catalyzed solvent-free reactions of thiazolidines $\mathbf{3}$ with aldehydes 2

\begin{tabular}{llllll}
\hline Entry & Aldehydes & Thiazolidenes & Product $^{\mathbf{a}}$ & Time (min) & Yield (\%) $^{\mathbf{b}}$ \\
\hline 1 & $2 a$ & $3 a$ & $\mathbf{4 a}$ & 20 & 95 \\
\hline 2 & $2 b$ & $3 a$ & $\mathbf{4 b}$ & 15 & 98 \\
\hline 3 & $2 c$ & $3 a$ & $\mathbf{4 c}$ & 25 & 91 \\
\hline 4 & $2 d$ & $3 a$ & $\mathbf{4 d}$ & 30 & 92 \\
\hline 5 & $2 e$ & $3 a$ & $\mathbf{4 e}$ & 20 & 96 \\
\hline 6 & $2 a$ & $3 b$ & $\mathbf{5 a}$ & 15 & 97 \\
\hline 7 & $2 b$ & $3 b$ & $\mathbf{5 b}$ & 10 & 99 \\
\hline 8 & $2 c$ & $3 b$ & $\mathbf{5 c}$ & 20 & 92 \\
\hline 9 & $2 d$ & $3 b$ & $\mathbf{5 d}$ & 25 & 91 \\
\hline 10 & $2 e$ & $3 b$ & $\mathbf{5 e}$ & 15 & 98 \\
\hline
\end{tabular}

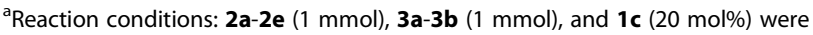
irradiated under ultrasound irradiations at $80^{\circ} \mathrm{C}$. The products were characterized by spectral techniques like IR, ${ }^{1} \mathrm{H} N \mathrm{NMR},{ }^{13} \mathrm{C} N \mathrm{NR}$, and mass spectra. ${ }^{b}$ Isolated yields after recrystallization.

minutes (see Table 3). The progress of the reaction was monitored via thin layer chromatography. After the reaction completion, the reaction mass was cooled $\left(15^{\circ} \mathrm{C}\right.$ to $20^{\circ} \mathrm{C}$ ) and stirred with water $(10 \mathrm{~mL})$ for $30 \mathrm{~min}$. The solid product was filtered and dried. The obtained products were recrystallized in EtOH/DMF (3:2). The products 4-5 were confirmed by their spectral data after comparison with authentic samples, infrared (IR), proton nuclear magnetic resonance (1H NMR), mass spectra, and melting points.

\section{General procedure for the recovery of [TMG][Lac]}

Further, the obtained filtrate was extracted with diethyl ether $(3 \times 10 \mathrm{~mL})$, and the aqueous layer was dried under pressure. IL as a colorless oil was obtained which was further reused to catalyze more reactions.

\section{Spectral data of reprehensive compounds}

The following are the spectral data for $\mathbf{4 a}, \mathbf{4 e}, \mathbf{5 b}$, and 5e:

- (4a): Mp. $249^{\circ} \mathrm{C}$ to $250^{\circ} \mathrm{C}$, IR $\left(\mathrm{KBr}, \mathrm{cm}^{-1}\right): 3393$, 1671, 1605, 1434, 1201; ${ }^{1} \mathrm{H}$ NMR $(300 \mathrm{MHz}$, DMSO- $\left.\mathrm{d}_{6}\right) \delta 3.09(\mathrm{~s}, 3 \mathrm{H}), 7.08(\mathrm{~d}, 2 \mathrm{H}, J=8.2 \mathrm{~Hz})$, $7.52(\mathrm{~d}, 2 \mathrm{H}, J=8.2 \mathrm{~Hz}), 7.61$ (s, 1H), 13.71 (s, 1H). Analysis calculated for $\mathrm{C}_{11} \mathrm{H}_{9} \mathrm{NO}_{2} \mathrm{~S}_{2}$ : C, 52.57\%; $\mathrm{H}$, 3.61\%; N, 5.57\%; S, 25.52\%; found: C, 52.78\%; H, $3.95 \% ;$ N, $5.48 \%$; S, $25.79 \%$

- (4e) Mp.: $259^{\circ} \mathrm{C}$ to $260^{\circ} \mathrm{C}$, IR $\left(\mathrm{KBr}, \mathrm{cm}^{-1}\right): 1647$ ( $\gamma$ pyrone $\mathrm{CO}) ;{ }^{1} \mathrm{H}$ NMR $\delta 7.54(\mathrm{ddd}, 1 \mathrm{H}, 6-\mathrm{H})$, $7.62(\mathrm{~s}, 1 \mathrm{H}, \mathrm{C}=\mathrm{C}-\mathrm{H}), 7.71\left(\mathrm{~d}, 1 \mathrm{H}, \mathrm{J}_{8,7}=8.41 \mathrm{~Hz}\right.$, 8-H), 7.98 (ddd, $1 \mathrm{H}, 7-\mathrm{H}), 8.17$ (dd, $1 \mathrm{H}$, $\left.J_{5,6}=8.41 \mathrm{~Hz}, J_{5,7}=1.68 \mathrm{~Hz}, 5-\mathrm{H}\right), 8.83(\mathrm{~s}, 1 \mathrm{H}$, 2-H), 12.41 (s, 1H, NH). Analysis calculated for $\mathrm{C}_{13} \mathrm{H}_{7} \mathrm{NO}_{3} \mathrm{~S}_{2}$ : C, 53.98\%; H, 2.42\%; N, 4.84\%;

S, 22.15\%; found: C, 53.84\%; H, 2.74\%; N, 4.95\%; S, $21.96 \%$

- (5b): Mp. $268^{\circ} \mathrm{C}$ to $269^{\circ} \mathrm{C}$. IR $\left(\mathrm{KBr}, \mathrm{cm}^{-1}\right)$ : 3148, 1719, 1610; ${ }^{1} \mathrm{H}$ NMR (300 MHz, DMSO-d $6 \delta_{\mathrm{H}}: 7.53(2 \mathrm{H}, \mathrm{m}), 7.72(2 \mathrm{H}, \mathrm{m}), 7.76$ $(1 \mathrm{H}, \mathrm{s}), 12.65$ (1H, bs). Analysis calculated for $\mathrm{C}_{10} \mathrm{H}_{6} \mathrm{ClNO}_{2} \mathrm{~S}$ : C, 50.11\%; H, 2.52\%; N, 5.84\%;

S, $13.38 \%$; found: C, 49.87\%; H, 2.63\%; N, 5.92\%; S, $13.78 \%$

- (5e) Mp.: $290^{\circ} \mathrm{C}$, IR (KBr, cm $\left.{ }^{-1}\right): 1637$ ( $\gamma$ pyrone $\mathrm{CO}) ;{ }^{1} \mathrm{H}$ NMR: $\delta 7.58(\mathrm{ddd}, 1 \mathrm{H}, 6-\mathrm{H})$, $7.61(\mathrm{~s}, 1 \mathrm{H}, \mathrm{C}=\mathrm{C}-\mathrm{H}), 7.74\left(\mathrm{~d}, 1 \mathrm{H}, J_{8,7}=8.40 \mathrm{~Hz}\right.$, $8-\mathrm{H}), 7.88$ (ddd, $1 \mathrm{H}, 7-\mathrm{H}), 8.13(\mathrm{dd}, 1 \mathrm{H}$, $\left.J_{5,6}=8.40 \mathrm{~Hz}, J_{5,7}=1.60 \mathrm{~Hz}, 5-\mathrm{H}\right), 8.85(\mathrm{~s}, 1 \mathrm{H}$, 2-H), 12.48 (s, 1H, NH). Analysis calculated for $\mathrm{C}_{13} \mathrm{H}_{7} \mathrm{NO}_{4} \mathrm{~S}$ : C 57.14\%, H 2.58\%, N 5.13\%, S 11.73\%; found: C 56.84\%, H 2.74\%, N 5.25\%, S $11.46 \%$

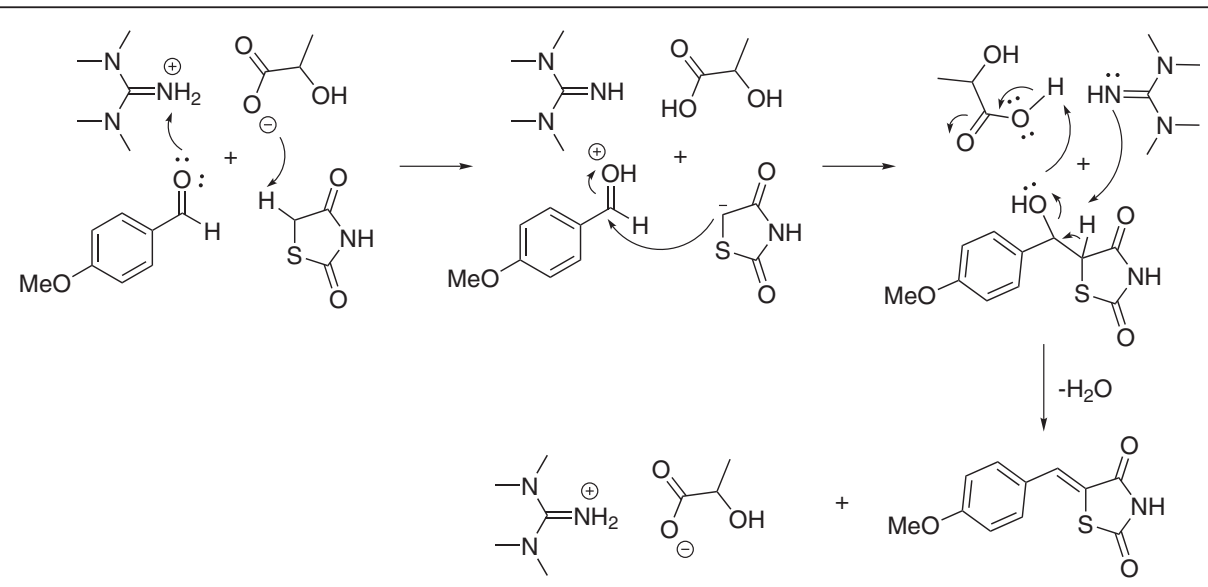

Scheme 2 The possible reaction mechanism for the Knoevenagel reaction catalyzed by TSIL. 


\section{Competing interests}

The authors declare that they have no competing interests.

\section{Acknowledgments}

The authors are also thankful to the Sophisticated Analytical Instrument Facility Central Instrument Laboratory (SAIF-CIL), Panjab University, Chandigarh for the spectral analysis.

\section{Received: 25 August 2012 Accepted: 6 February 2013} Published: 3 March 2013

\section{References}

1. Carroll RT, Dluzen DE, Stinnet H, Awale PS, Funk MO, Geldenhuys WJ (2011) Structure-activity relationship and docking studies of thiazolidinedione-type compounds with monoamine oxidase B. Bioorg Med Chem Lett 21:4798-4803. doi:10.1016/j.bmcl.2011.06.060

2. Bruno G, Costantino L, Curinga C, Maccari R, Monforte F, Nicolò F, Ottanà R, Vigorita MG (2002) Synthesis and aldose reductase inhibitory activity of 5-arylidene-2,4-thiazolidinediones. Bioorg Med Chem 10:1077-1084. http://dx.doi.org/10.1016/S0968-0896(01)00366-2

3. Diurno MV, Mazzoni O, Correale G, Monterrey IG, Calignano A, Rana GL, Bolognese A (1999) Synthesis and structure-activity relationships of 2(substituted phenyl)-3-3-(N,N-dimethylamino)propyl-1,3-thiazolidin-4-ones acting as H1-histamine antagonists. IL Farmaco 54:579-583. http://dx.doi.org/10.1016/S0014-827X(99)00064-6

4. Ergenc N, Capan G (1994) Synthesis and anticonvulsant activity of new 4-thiazolidone and 4-thiazoline derivatives. IL Farmaco 49:133-135

5. Piscapo E, Diurno MV, Gagliardi R, Mazzoni O (1989) Studies on heterocyclic compounds: 1,3-thiazolidin-4-one derivatives. IV. Biological activity of variously substituted 2,3-diaryl-1,3-thiazolidin-4-ones. Boll Soc Ital Niol Sper 65:853-859

6. Previtera T, Vigorita MG, Bisila M, Orsini F, Benetolla F, Bombieri G () 3,3'-di1,3-thiazolidine-4-one.system. VI: Structural and conformational studies on configurational isomers with antihistaminic activity. Eur J Med Chem 29:317-324. http://dx.doi.org/10.1016/0223-5234(94)90102-3

7. Havrylyuka D, Zimenkovskya B, Lesyka R (2009) Synthesis and anticancer activity of novel nonfused bicyclic thiazolidinone derivatives. Phosphorus, sulfur, and silicon and the related elements. 184:638-650. doi:10.1080/ 10426500802247563

8. Rawal RK, Prabhakar YS, Katti SB, De Clercq E (2005) 2-(Aryl)-3-furan-2ylmethyl-thiazolidin-4-ones as selective HIV-RT inhibitors. Bioorg Med Chem 13:6771-6776. http://dx.doi.org/10.1016/j.bmc.2005.07.063

9. Wu Y, Karna S, Choi CH, Tong M, Tai HH, Na DH, Jang CH, Cho H (2011) Synthesis and biological evaluation of novel thiazolidinedione analogues as 15-hydroxyprostaglandin dehydrogenase inhibitors. J Med Chem 54:5260-5264. doi:10.1021/jm200390u

10. Adachi Y, Suzuki Y, Homma N, Fukazawa M, Tamura K, Nishie I, Kuromaru O (1999) The anti-ischemic effects of CP-060S during pacing-induced ischemia in anesthetized dogs. Eur J Pharmacol 367:267-273. http://dx.doi.org/ 10.1016/S0014-2999(98)00938-8

11. Safonov IG, Heerding DA, Keenan RM, Price AT, Erickson-Muller CL, Hopson CB, Levin JL, Lord KA, Tapley PM (2006) New benzimidazoles as thrombopoietin receptor agonists. Biorg Med Chem Lett 16:1212-1216. http://dx.doi.org/10.1016/j.bmcl.2005.11.096

12. Lohray BB, Bhushan V, Rao PB, Madhavan GR, Murali N, Rao KN, Reddy KA, Rajesh BM, Reddy PG, Chakrabarti R, Rajagopalan R (1997) Novel indole containing thiazolidinedione derivatives as potent euglycemic and hypolipidaemic agents. Bioorg Med Chem Lett 7:785-788. http://dx.doi.org/10.1016/S0960-894X(97)00118-2

13. Zhou JF, Zhu FX, Song YZ, Zhu YL (2006) Synthesis of 5arylalkylidenerhodanines catalyzed by tetrabutylammonium bromine in water under microwave irradiation. Arkivoc 14:175-180

14. Pratap UR, Jawale DV, Waghmare RA, Lingampalle DL, Mane RA (2011) Synthesis of 5-arylidene-2,4-thiazolidinediones by Knoevenagel condensation catalyzed by baker's yeast. New J Chem 35:49-51. doi:10.1039/CONJ00691B

15. Powers JP, Piper DE, Li Y, Mayorga V, Anzola J, Chen JM, Jaen JC, Lee G, Liu J, Peterson MG, Tonn GR, Ye QY, Walker NPC, Wang Z (2006) SAR and mode of action of novel non-nucleoside inhibitors of hepatitis C NS5b RNA polymerase. J Med Chem 49:1034-1046. doi:10.1021/jm050859x
16. Wrobleski ML, Reichard GA, Paliwal S, Shah S, Tsui HC, Duffy RA, Lachowicz JE, Morgan CA, Varty GB, Shih NY (2006) Cyclobutane derivatives as potent NK1 selective antagonists. Bioorg Med Chem Lett 16:3859-3863. http://dx.doi.org/10.1016/j.bmcl.2006.04.031

17. Song Y, Connor DT, Doubleday R, Sorenson RJ, Sercel AD, Unangst PC, Roth BD, Gilbertsen RB, Chan K, Schrier DJ, Guglietta A, Bornemeier DA, Dyer RD (1999) Synthesis, structure-activity relationships, and in vivo evaluations of substituted di-tert-butylphenols as a novel class of potent, selective, and orally active cyclooxygenase-2 inhibitors. 1. Thiazolone and oxazolone series. J Med Chem 42:1151-1160. doi:10.1021/jm9805081

18. Chowdhy MM, Michael D, Mingas P, White AJP, William DJ (2000) Syntheses and characterization of 5-substituted hydantoins and thiazolinesimplications for crystal engineering of hydrogen bonded assemblies. Crystal structures of 5-(2- pyridylmethylene)hydantoin, 5-(2pyridylmethylene)-2-thiohydantoin, 5-(2-pyridylmethylene) thiazolidine-2,4dione, 5-(2-pyridylmethylene) rhodanine and 5-(2-pyridylmethylene) pseudothiohydantoin. J Chem Soc Perkin Trans 1:3495-3504. doi:10.1039/B004312P

19. Yang BY, Yang DH (2011) Solvent-free synthesis of 5-benzylidene-2thioxothiazolidin-4-ones and thiazolidine-2,4-diones catalysed by glycine under microwave irradiation. J Chem Res 4:238-239. doi:10.3184/ $174751911 \times 13025104502362$

20. Yang DH, Yang BY, Chen BC, Chen SYA (2006) Convenient synthesis of 5-arylidenethiazolidine-2,4-diones on potassium fluoride-aluminium oxide. Org Prep Pro Int 38:81-85. doi:10.1080/00304940609355982

21. Alizadeh A, Khodaei MM, Eshghi A (2010) A solvent-free protocol for the green synthesis of arylalkylidenerhodanines in a task-specific ionic liquid. Can J Chem 88:514-518

22. Jawale DV, Pratap UR, Lingampalle DL, Mane RA (2011) Dicationic ionic liquid mediated synthesis of 5-arylidine-2,4-thiazolidinediones. Chin J Chem 29:942-946. doi:10.1002/cjoc.201190192

23. Gong K, He ZW, Xu Y, Fang D, Liu Z-L (2008) Synthesis of 5-benzylidene rhodanine derivatives catalyzed by 1-butyl-3-methyl imidazolium hydroxide in water. Monatsh Chem 139:913-915. doi:10.1007/s00706-008-0871-y

24. Shelke KF, Sapkal SB, Madje BR, Shingate BB, Shingare MS (2009) Ionic liquid promoted an efficient synthesis of 5-arylidene-2,4-thiazolidinedione. Bull Catal Soc India 8:30-34

25. Yang D-H, Chen Z-C, Chen S-Y, Zheng Q-G (2003) Organic reactions in ionic liquids; ionic liquid-accelerated three-component reaction: a rapid one-pot synthesis of 3-alkyl-5-(Z)-arylmethylidene-1,3-thiazolidine-2,4-diones 1891-1894. Synthesis. doi:10.1055/s-2003-40980

26. Welton $T$ (1999) Room-temperature ionic liquids: solvents for synthesis and catalysis. Chem Rev 99:2071-2084. doi:10.1021/cr980032t

27. Wasserscheid P, Keim W (2000) lonic liquids-new "Solutions" for transition metal catalysis. Angew Chem Int Ed 39:3772-3789. doi:10.1002/1521-3773 (20001103)39:21<3772::AID-ANIE3772>3.0.CO;2-5

28. Dupont J, de Souza RF, Suarez PAZ (2002) lonic liquid (molten salt) phase organometallic catalysis. Chem Rev 102:3667-3691. doi:10.1021/cr010338r

29. Suresh SJS (2011) Recent advances in ionic liquids: green unconventional solvents of this century Part I. Green Chem Lett Rev 4:289-310. doi:10.1080/17518253.2011.572294

30. Suresh SJS (2011) Recent advances in ionic liquids: Green unconventional solvents of this century Part II. Green Chem Lett Rev 4:311-320. doi:10.1080/17518253.2011.572295

31. Gao H, Han B, Li J, Jiang T, Liu Z, Wu W, Chang Y, Zhang J (2004) Preparation of room-temperature ionic liquids by neutralization of 1,1,3, 3-tetramethylguanidine with acids and their use as media for Mannich reaction. Synth Commun 34:3083-3089. doi:10.1081/SCC-200028532

32. Jiang T, Gao H, Han B, Zhao G, Chang Y, Wu W, Gao L, Yang G (2004) lonic liquid catalyzed Henry reactions. Tetrahedron Lett 45:2699-2701. doi:10.1016/j.tetlet.2004.01.129

33. Huang J, Jiang T, Gao H, Han B, Liu Z, Wu W, Chang Y, Zhao G (2004) Pd nanoparticles immobilized on molecular sieves by ionic liquids: heterogeneous catalysts for solvent-free hydrogenation. Angew Chem Int Ed 43:1397-1399. doi:10.1002/ange.200352682

34. Jiang T, Zhou Y, Liang S, Liu H, Han B (2009) Hydrogenolysis of glycerol catalyzed by Ru-Cu bimetallic catalysts supported on clay with the aid of ionic liquids. Green Chem 11:1000-1006. doi:10.1039/B901425J

35. Zhu A, Jiang T, Han B, Huang J, Zhang J, Ma X (2006) Study on guanidinebased task-specific ionic liquids as catalysts for direct aldol reactions without solvent. New J Chem 30:736-740. doi:10.1039/B600277C 
36. Zhu A, Jiang T, Wang D, Han B, Liu L, Huang J, Zhang J, Sun D (2005) Direct aldol reactions catalyzed by 1,1,3,3-tetramethylguanidine lactate without solvent. Green Chem 7:514-517. doi:10.1039/B501925G

37. Chavan SS, Degani MS (2011) Ionic liquid catalyzed 4,6-disubstituted-3cyano-2-pyridone synthesis under solvent-free conditions. Catal Lett 141:1693-1697. doi:10.1007/s10562-011-0700-5

38. Liang S, Liu H, Zhou Y, Jiang T, Han B (2010) The tetramethylguanidinebased ionic liquid-catalyzed synthesis of propylene glycol methyl ether. New J Chem 34:2534-2536. doi:10.1039/CONJ00502A

39. Li JT, Wang SX, Chen GF, Li TS (2005) Some applications of ultrasound irradiation in organic synthesis. Curr Org Synth 2:415-436. doi:10.1002/ chin.200541232

40. Cravotto G, Cintas P (2006) Power ultrasound in organic synthesis: moving cavitational chemistry from academia to innovative and large-scale applications. Chem Soc Rev 35:180-196. doi:10.1039/B503848K

41. Cella R, Stefani H (2009) Ultrasound in heterocycles chemistry. Tetrahedron 65:2619-2641. http://dx.doi.org/10.1016/j.tet.2008.12.027

doi:10.1186/2191-2858-3-2

Cite this article as: Suresh and Sandhu: Ultrasound-assisted synthesis of 2,4-thiazolidinedione and rhodanine derivatives catalyzed by taskspecific ionic liquid: [TMG][Lac]. Organic and Medicinal Chemistry Letters 2013 3:2.

\section{Submit your manuscript to a SpringerOpen ${ }^{\circ}$ journal and benefit from:}

- Convenient online submission

- Rigorous peer review

- Immediate publication on acceptance

- Open access: articles freely available online

- High visibility within the field

- Retaining the copyright to your article 